\title{
Hydromagnetic rotating flow in a porous medium with slip condition and Hall current
}

\author{
A. Farhad, M. Norzieha*, S. Sharidan, I. Khan and Samiulhaq \\ Department of Mathematics, Faculty of Science, Universiti Teknologi Malaysia, 81310 UTM Skudai, Johor Malaysia.
}

Accepted 26 January, 2012

The slip effect on hydromagnetic rotating flow of viscous fluid through a porous space is investigated. The fluid is electrically conducting with the consideration of Hall current. The entire system rotates about the axis normal to a porous plate of uniform suction or injection with uniform angular velocity. The closed form solution is obtained using Laplace transform technique. The analytical expression for skin friction is evaluated. The graphical results are displayed to see the effects of various embedded flow parameters such as magnetic parameter $M$, permeability parameter $K$, Hall parameter $m$, rotation parameter $\eta$, suction or injection parameter $S$, slip parameter $\gamma$ and dimensionless time $\tau$. It is found that the magnetic field and slip parameter decrease the velocity magnitude whereas permeability and Hall parameters increase it. The slip and magnetic field play an important role in retarding the growth of both the primary and secondary flows, whereas Hall parameter enhances the flow.

Key words: Slip effect, rotating flow, porous space, laplace transform.

\section{INTRODUCTION}

Although, the rotating flow of viscous fluid is useful, familiar and well-studied in the literature (Hayat et al., 2008a, 2008b, Hayat and Nawaz, 2011a; Asghar et al., 2007; Turkyilmazoglu, 2009; Abelman et al., 2009; Chawla et al., 2009; Singh et al., 2009; Sahoo et al., 2010; Seth et al., 2010; Jana et al., 2010), yet, the underlying physics is surprisingly subtle and complex. The behavior of fluids under extreme confinement is of great interest from both the scientific and the technological points of view. One of the great complexity is to discover what type of boundary conditions are appropriate for solving the continuum fluid problems. Despite of the widespread acceptance of no slip assumption, there has been existed for many years, indirect experimental evidence based on anomalous flow in capillaries and other systems that in some cases, simple liquids can slip against solids when walls are sufficiently smooth and the

\footnotetext{
*Corresponding author. E-mail: norzieha@utm.my.
}

no slip boundary condition is no more valid. The no-slip boundary condition is valid only when particles close to a surface do not move along with a flow when adhesion is stronger than cohesion. However, this is only true macroscopically. Few other limitations of no-slip condition are: fails for large contact angles, does not hold at a very low pressure, does not work for polyethylene, rubber compounds and suspensions, fails in hydrodynamics for hydrophobic surfaces (Vinogradova, 1995; Zhu and Granick, 2002). Recently, the slip condition has become much more compelling and it is now reasonably certain that viscous fluids can slip against solid surfaces if the fluid does not fully wet the solid surface or the solid surface is very smooth (Navier, 1823). The slip boundary condition has significant applications in lubrication, extrusion, medical sciences, especially in polishing artificial heart valves, flows through porous media, micro and nanofluidics, friction studies and biological fluids (Blake, 1990; Pit et al., 1999).

The influence of a transverse magnetic field and radiative heat transfer on the unsteady oscillatory flow of 
a viscous fluid in a channel filled with porous medium was studied by Makinde and Mhone (2005). Mehmood and Ali (2007) extended the work of Makinde and Mhone (2005) by considering the slip effect at the boundary and obtained the analytical results for velocity and temperature distributions. Recently, Qasim et al. (2011) studied the effects of slip conditions on stretching flow with ohmic dissipation and thermal radiation. The influence of slip condition on the MHD flow and heat transfer over permeable stretching sheet is investigated by Hayat et al. (2011b). Hayat et al. (2010a, 2010b) analyzed the influence of slip condition on the rotating and magnetohydrodynamics (MHD) Couette flow of an Oldroyd-B fluid and generalized Burgers' fluid through a porous space. Furthermore, the study of MHD flow with Hall current has important applications in problems of Hall accelerators, flight magnetohydrodynamics, in the dynamics of the fluid and the magnetic fields of many astrophysical objects. The influence of Hall current on the rotating oscillating flows of an Oldroyd-B fluid in a porous medium has been studied by Hayat et al. (2007). In few other investigations (Katagiri, 1962; Jana et al., 1977; Gosh, 1993, 1999) have discussed the MHD flow under different physical aspects, whereas, Mandal and Mandal (1983) and Jha and Apere (2010) have studied the effect of Hall currents under varying conditions. To the best of our knowledge, the effect of slip condition with Hall current on the magnetohydrodynamic flow of a rotating viscous fluid in a porous medium bounded by a porous plate has not been discussed in the literature of rotating flows. Therefore, this is the basic objective of the present article. The problem is first modeled and then solved for the closed form solution by using Laplace transform technique.

The expression for velocity field has been obtained in term of complementary error function and discussed in detail with the help of graphs for various embedded flow parameters. The analytical expression for the skin friction has been computed from the complex velocity field.

\section{MATHEMATICAL FORMULATION OF THE PROBLEM}

This analysis considers the unsteady flow of an incompressible viscous fluid over an infinitely extended porous plate resting at $z=0$. The positive $z-$ axis is taken normal to the plate and $x-$ axis parallel to it. The fluid occupies the porous space of $z>0$ and is assumed to be electrically conducting under the influence of a uniform magnetic field BO applied in a direction parallel to the zaxis. The induced magnetic field is neglected for small magnetic Reynolds number while the effect of Hall current is considered. It is assumed that the whole system is in the state of rigid body rotation normal to a porous plate of uniform suction $w_{0}>0$ or injection $w_{0}<0$ with constant angular velocity $\boldsymbol{\Omega}=\Omega \hat{\mathbf{k}} \quad(\hat{\mathbf{k}} \quad$ is a unit vector in the $z$-direction). The plate at $z=0$ is suddenly moved for $t=0^{+}$. Under these assumptions, the flow is governed by the following continuity and momentum equations: $\operatorname{div} \mathbf{V}=0$

$\rho\left(\frac{\partial \mathbf{V}}{\partial t}+(\mathbf{V} \cdot \boldsymbol{\nabla}) \mathbf{V}+\mathbf{2} \boldsymbol{\Omega} \times \mathbf{V}+\boldsymbol{\Omega} \times(\boldsymbol{\Omega} \times \mathbf{r})\right)=\operatorname{div} \mathbf{T}+\mathbf{J} \times \mathbf{B}+\mathbf{R},(2)$

Where $\mathbf{V}=(u, v, w)$ is the velocity vector, $\rho$ is the fluid density, $\nabla$ is the gradient operator, $\mathbf{R}$ is the Darcy's resistance, $\mathbf{r}$ is a radial vector with $r^{2}=x^{2}+y^{2}$. The Cauchy stress tensor $\mathbf{T}$ for an incompressible viscous fluid is given as:

$\mathbf{T}=-p \mathbf{I}+\mu \mathbf{A}_{1}$,

in which $p \mathbf{I}$ is the indeterminate spherical stress, $\mu$ is the dynamic viscosity and $\mathbf{A}_{1}$ is the first Rivilin-Ericken tensor given as:

$\mathbf{A}_{1}=\mathbf{L}+\mathbf{L}^{\mathrm{T}}$

Where $\mathbf{L}=\operatorname{grad} \mathbf{V}$ and $\mathrm{T}$ indicates the matrix transpose. The velocity field is assumed of the following form:

$\mathbf{V}=\left(u(z, t), v(z, t), w_{0}\right)$

Where $t$ is the time, $u$, and $v$ are the velocity components in $x$, and $y-$ directions and $w_{0}$ is suction or injection velocity. The Darcy's resistance for viscous fluid is given as:

$\mathbf{R}=-\frac{\mu \phi}{k} u$

in which $\phi(0<\phi<1)$ is the porosity and $k>0$ is the permeability of the porous medium. Taking into account the Hall effect and considering the Maxwell's equations:

$\operatorname{div} \mathbf{B}=0, \quad \operatorname{curl} \mathbf{B}=\mu_{m} \mathbf{J}, \quad \operatorname{curl} \mathbf{E}=-\frac{\partial \mathbf{B}}{\partial t}$,

the usual Ohm's law modifies to Gosh (1999):

$\mathbf{J}+\frac{\omega_{e} \tau_{e}}{B_{0}}(\mathbf{J} \times \mathbf{B})=\sigma\left[\mathbf{E}+\mathbf{V} \times \mathbf{B}+\frac{1}{e n_{e}} \nabla p_{e}\right]$

called the generalized Ohm's law. Here, $\mathbf{B}$ is the total magnetic field, $B_{0}$ is the strength of applied magnetic field parallel to $z-$ axis, $\mathbf{J}$ is the current density, $\mathbf{E}$ is the total electric field, $\mu_{m}$ is the magnetic permeability, $\omega_{e}$ is the cyclotron frequency, $\tau_{e}$ is the electron collision time, $\sigma$ is the electrical conductivity of the fluid, $e$ is the electron charge, $p_{e}$ is the electron pressure and $n_{e}$ is the number density of electrons. For weakly ionized gases, we neglect Equation 8, the electron pressure gradient, the ion-slip and the thermo-electric effects. 
Inserting equation (5), the continuity equation (1) is automatically satisfied and the momentum (Equation 2) in view of Equations 3, 4, 6 to 8 , yields:

$\frac{\partial F}{\partial t}-w_{0} \frac{\partial F}{\partial z}+2 i \Omega F=v \frac{\partial^{2} F}{\partial z^{2}}-\frac{v \phi}{k} F-\frac{\sigma B_{0}^{2}}{\rho(1-i m)} F, \quad z, \quad t>0,(9)$

where $F=u+i v$ is the complex fluid velocity and $m=\omega_{e} \tau_{e}, \quad$ is the Hall parameter. The initial and boundary conditions relevant to the problem are:

$F(z, 0)=0 ; \quad t \leq 0$,

$F(0, t)-\lambda \frac{\partial F}{\partial z}(0, t)=U_{0} ; \quad t>0$,

$F(z, t) \rightarrow 0 ;$ as $z \rightarrow \infty ; \quad t>0$,

Where $\lambda$ is the slip parameter.
We now introduce the following dimensionless variables (Jana et al., 2010):

$$
G=\frac{F}{U_{0}}, \quad \xi=\frac{z U_{0}}{v}, \quad \tau=\frac{U_{0}^{2} t}{v},
$$

and substitute into Equations 9 to 11, the dimensionless equation takes the following form:

$\frac{\partial G}{\partial \tau}-S \frac{\partial G}{\partial \xi}+2 i \eta G=\frac{\partial^{2} G}{\partial \xi^{2}}-\frac{M^{2}}{1-i m} G-\frac{1}{K} G$

Where the boundary and initial conditions become:

$$
\begin{aligned}
& G(\xi, 0)=0 ; \quad \tau \leq 0, \\
& G(0, \tau)-\gamma \frac{\partial G}{\partial \xi}(0, \tau)=1 ; \quad \tau>0, \\
& G(\infty, \tau)=0 ; \quad \tau>0,
\end{aligned}
$$

With the following dimensionless parameters:

$$
\begin{gathered}
M^{2}=\frac{\sigma B_{0}^{2} v}{\rho U_{0}^{2}}(\text { Hartmann number }), \frac{1}{K}=\frac{v^{2} \phi}{k U_{0}^{2}}(\text { Porosity } \mathrm{p} \text { arameter }) \\
\eta=\frac{\Omega v}{U_{0}^{2}} \text { (Rotation parameter), } S=\frac{w_{0}}{U_{0}}(\text { Suction parameter }) \text { and } \\
\gamma=\frac{\lambda U_{0}}{v} \quad \text { (Dimensionless lip parameter) }
\end{gathered}
$$

\section{SOLUTION OF THE PROBLEM}

Taking the Laplace transform of Equation 14, using initial condition (Equation 15), we get:

$\frac{d^{2} \bar{G}(\xi, q)}{d \xi^{2}}+S \frac{d \bar{G}(\xi, q)}{d \xi}-\left[q+\frac{M^{2}}{1-i m}+\frac{1}{K}+2 i \eta\right] \bar{G}(\xi, q)=0$.

The corresponding boundary conditions (Equations 16 and 17) are transformed into:

$$
\begin{aligned}
\bar{G}(\mathbf{O}, q)-\gamma \frac{d \bar{G}(\mathbf{O}, q)}{d \xi} & =\frac{\mathbf{1}}{q}, \\
G(\xi, \tau)= & -\frac{e^{-\xi\left(-\frac{s}{2}+A\right)} e^{-i B \xi}}{2-\gamma S+2 \gamma(A+i B)} \\
& -\frac{4 \gamma e^{\frac{\xi}{2}}}{(2-\gamma S)^{2}-4 \gamma^{2} L}\left[\frac{e^{-\frac{\xi^{2}}{4 \tau}-L \tau}}{\sqrt{\pi \tau}}-\mathrm{d} e^{\xi \mathrm{d}+\mathrm{d}^{2} \tau-L \tau} \operatorname{erf~} \mathrm{c}\left(\frac{\xi}{2 \sqrt{\tau}}+\mathrm{d} \sqrt{\tau}\right)\right] \\
& +\frac{e^{\xi \frac{\xi}{2}}}{\sqrt{L}(2+2 \gamma \sqrt{L}-\gamma S)}\left[\frac{e^{\frac{-\xi^{2}}{4 \tau}-L \tau}}{\sqrt{\pi \tau}}+\sqrt{L} e^{-\xi \sqrt{L}} \operatorname{erf~}\left(\sqrt{L \tau}-\frac{\xi}{2 \sqrt{\tau}}\right)\right] \\
& +\frac{e^{\xi \frac{\xi}{2}}}{\sqrt{L}(2-2 \gamma \sqrt{L}-\gamma S)}\left[\frac{e^{\frac{\xi^{2}}{4 \tau}-L \tau}}{\sqrt{\pi \tau}}-\sqrt{L} e^{\xi \sqrt{L}} \operatorname{erf~c}\left(\frac{\xi}{2 \sqrt{\tau}}+\sqrt{L \tau}\right)\right],
\end{aligned}
$$

Using boundary conditions (Equations 19 and 20), the solution of Equation 18 in the transformed $(\xi, q)$ plane is given by:

$$
\bar{G}(\xi, q)=\frac{1}{\gamma q(d+\sqrt{L+q})} \exp \left(-\xi\left(\frac{-S}{2}+\sqrt{L+q}\right)\right)
$$

The Laplace inverse of Equation 21 is obtained as follows in Equation 22 (Asghar et al., 2007): 
With

$$
\begin{aligned}
& L=\frac{S^{2}}{4}+\frac{1}{K}+\frac{M^{2}}{1-i m}+2 i \eta, \mathrm{d}=-\frac{S}{2}+\frac{1}{\gamma}, \\
& A=\frac{1}{\sqrt{2}} \sqrt{\sqrt{\left(\frac{S^{2}}{4}+\frac{1}{K}-\frac{M m}{1+m^{2}}\right)^{2}+\left(\frac{m^{2}}{1+m^{2}}+2 \eta\right)^{2}+\frac{S^{2}}{4}+\frac{1}{K}-\frac{M m}{1+m^{2}}}} \\
& B=\frac{1}{\sqrt{2}} \sqrt{\sqrt{\left(\frac{S^{2}}{4}+\frac{1}{K}-\frac{M m}{1+m^{2}}\right)^{2}+\left(\frac{m^{2}}{1+m^{2}}+2 \eta\right)^{2}}-\left(\frac{S^{2}}{4}+\frac{1}{K}-\frac{M m}{1+m^{2}}\right)} .
\end{aligned}
$$

Where erf $c(\cdot)$ denotes the complementary error function.

The dimensionless expression for the skin friction is given by:

$$
\begin{aligned}
\tau_{2}=-\frac{\partial G(0, \tau)}{\partial \xi} & (23) \\
\tau_{2}= & \frac{i B}{2+2(A+i B) \gamma-S \gamma}+\frac{-A+\frac{S}{2}}{2+2(A+i B) \gamma-S \gamma} \\
& +\frac{2 S \gamma\left(\frac{e^{-L \tau}}{\sqrt{\pi \tau}}-d \operatorname{Exp}\left(d^{2} \tau-L \tau\right) \operatorname{erf} \mathrm{c}(d \sqrt{\tau})\right)}{(2-\gamma S)^{2}-4 L \gamma^{2}}+\frac{4 \gamma\left(\frac{d e^{-L \tau}}{\sqrt{\pi \tau}}-d^{2} \operatorname{Exp}\left(d^{2} \tau-L \tau\right) \operatorname{erf} \mathrm{c}(d \sqrt{\tau})\right)}{(2-\gamma S)^{2}-4 L \gamma^{2}} \\
& -\frac{S\left(\frac{e^{-L \tau}}{\sqrt{\pi \tau}}-\sqrt{L} \operatorname{erf} \mathrm{c}(\sqrt{L \tau})\right)}{2 \sqrt{L}(2-2 \sqrt{L} \gamma-\gamma S)}-\frac{S\left(\frac{e^{-L \tau}}{\sqrt{\pi \tau}}+\sqrt{L} \operatorname{erf}(\sqrt{L \tau})\right)}{2 \sqrt{L}(2+2 \sqrt{L} \gamma-\gamma S)} \\
& -\frac{\frac{e^{-L \tau} \sqrt{L}}{\sqrt{\pi \tau}}-L \operatorname{erf} \mathrm{c}(\sqrt{L \tau})}{\sqrt{L}(2-2 \sqrt{L} \gamma-\gamma S)}-\frac{\frac{e^{-L \tau} \sqrt{L}}{\sqrt{\pi \tau}}-L \operatorname{erf} \mathrm{c}(\sqrt{L \tau})}{\sqrt{L}(2+2 \sqrt{L} \gamma-\gamma S)} .
\end{aligned}
$$

It is important to note that equation (22) satisfy the imposed initial and boundary conditions. Further, if we take $M=\gamma=0$, the solutions (Equations 22 and 24) reduce to those obtained by Jana et al. (2010), Equations 16 and 17 which provides a useful mathematical check.

\section{RESULTS AND DISCUSSION}

The problem of unsteady rotating hydromagnetic flow past a suddenly moved plate in a porous space with slip effect and uniform suction or injection has been studied. The closed form solution for the complex velocity in terms of complementary error function has been obtained by using Laplace transform technique. The numerical computations have been carried out for the nondimensional complex velocity for different values of the involved parameters and displayed graphically. The effects of magnetic parameter $M$, permeability parameter $K$, Hall parameter $m$, rotation parameter $\eta$,
Where $\tau_{2}=\frac{\tau_{1} v}{\mu U_{0}}$, and $\tau_{1}$ is the dimensional skin friction. Hence, Equation 23, using Equation 22 gives: suction or injection parameter $S$, slip parameter $\gamma$ and time $\tau$ have been observed on complex velocity. The behavior of these parameters on the velocity has been shown in Figures 1 to 8 . From these figures, the real and imaginary parts of the complex velocity are shown in panels $(a)$ and $(b)$ respectively. Figure 1 illustrates the variations of magnetic parameter $M$ on the real and imaginary parts of velocity. We observed a decrease in both real and imaginary parts of the complex velocity as well as the boundary layer thickness when the magnetic parameter $M$ is increased. As expected due to the fact that the application of transverse magnetic field results to a resistive type force (called Lorentz force) similar to drag force and upon increasing the values of $M$, the drag force increases which leads to the deceleration of the flow. In Figure 2, the profiles of velocity versus $\xi$ have been plotted for various values of permeability parameter $K$ by keeping other parameters fixed. Clearly, for large 


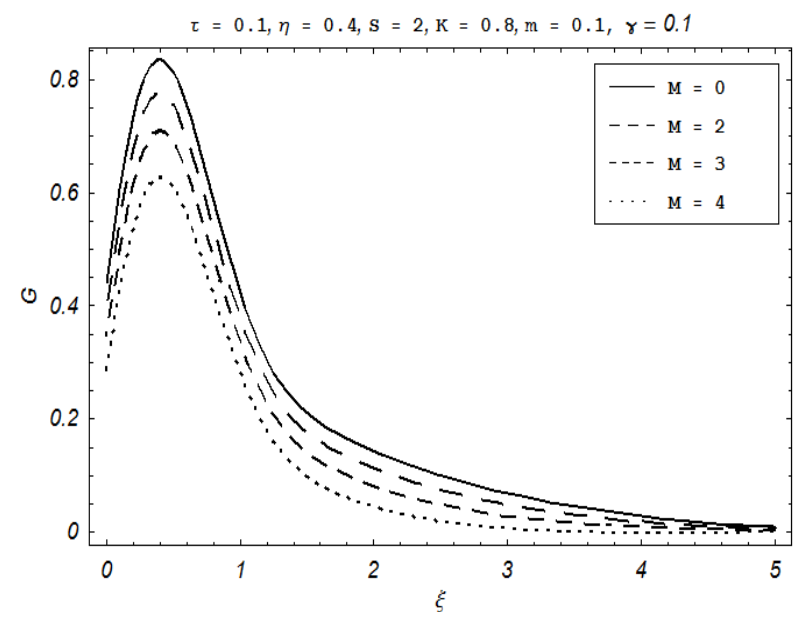

(a)

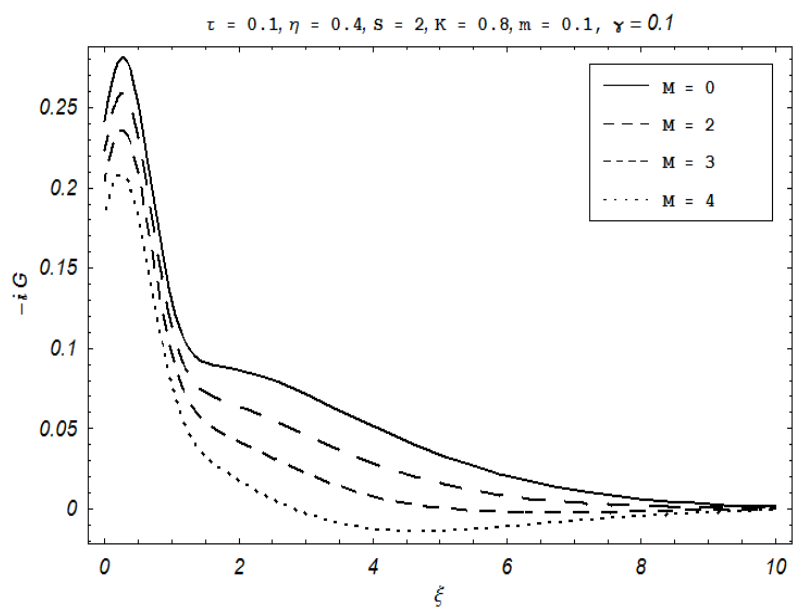

(b)

Figure 1. Profiles of velocity for different values of $M$.

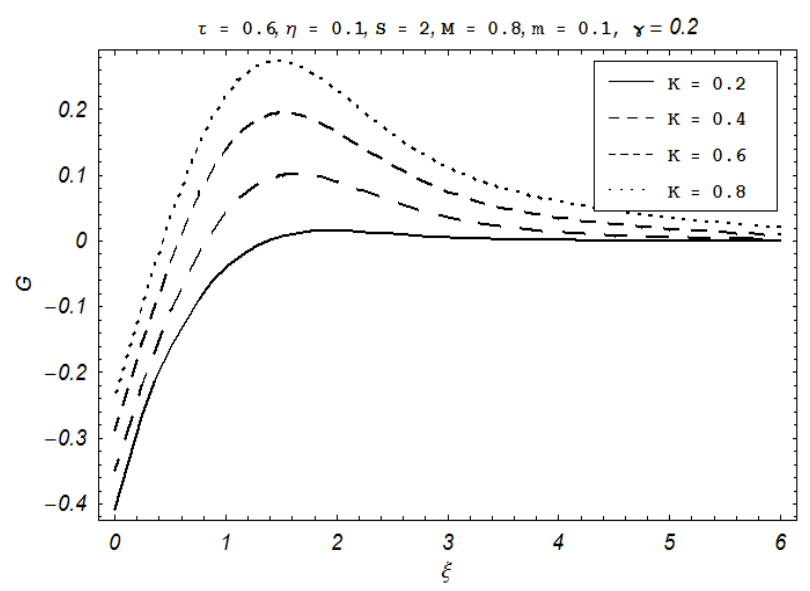

(a)

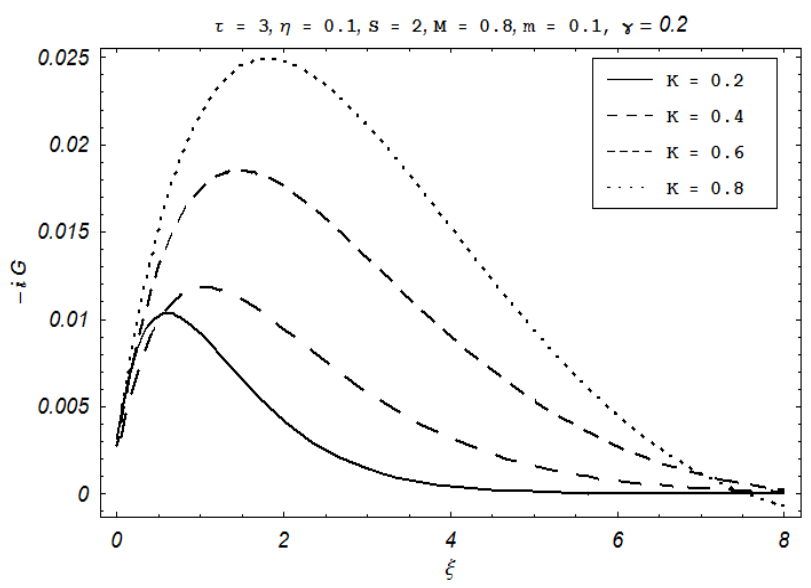

(b)

Figure 2. Profiles of velocity for different values of $K$.

values of $K$, both real and imaginary parts of velocity and the boundary layer thickness is increasing. However, further inspection reveals that increase in boundary layer thickness for the imaginary part of velocity is greater compared to the real part of velocity. This explains the fact that as $K$ increases; the resistance of the porous medium decreases which increases the momentum development of the flow regime ultimately increases the velocity field. The effect of $m$ on the complex velocity field is shown in Figure 3. We observed that the variation of velocity with increasing values of $m$ is similar to that of $K$. In Figure 4, the velocity profiles for complex velocity $G$ versus $\xi$ have been plotted for various values of rotation parameter $\eta$ on the complex velocity. We observed that the real part of the velocity is decreasing whereas the magnitude of imaginary part is increasing for large values of $\eta$.

The solid curves in panels $a$ and $b$ represent the fluid velocity when there is no rotation. Figures 5 and 6 depict the velocity distribution versus span wise coordinate $\xi$. For different values of suction or injection parameter $S$ respectively. From Figure 5, it is observed that, when 


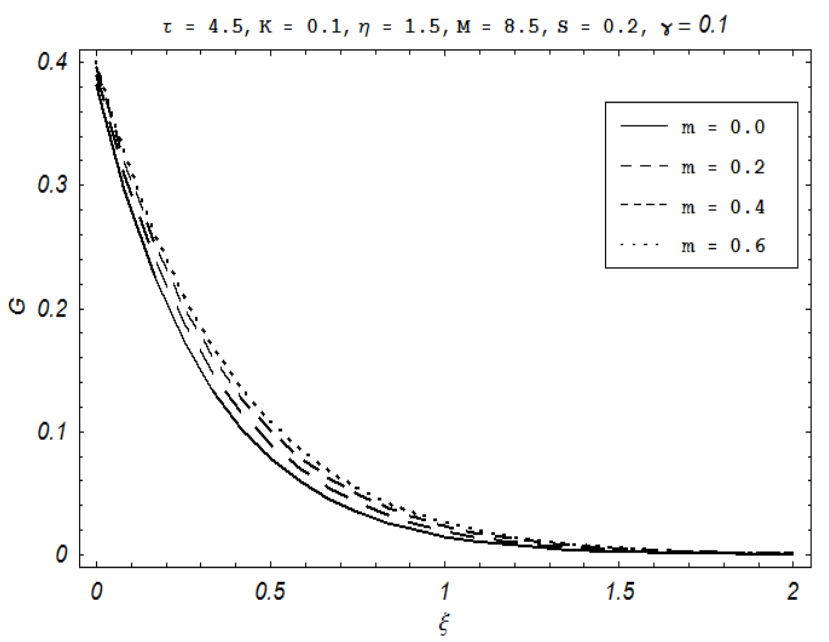

(a)

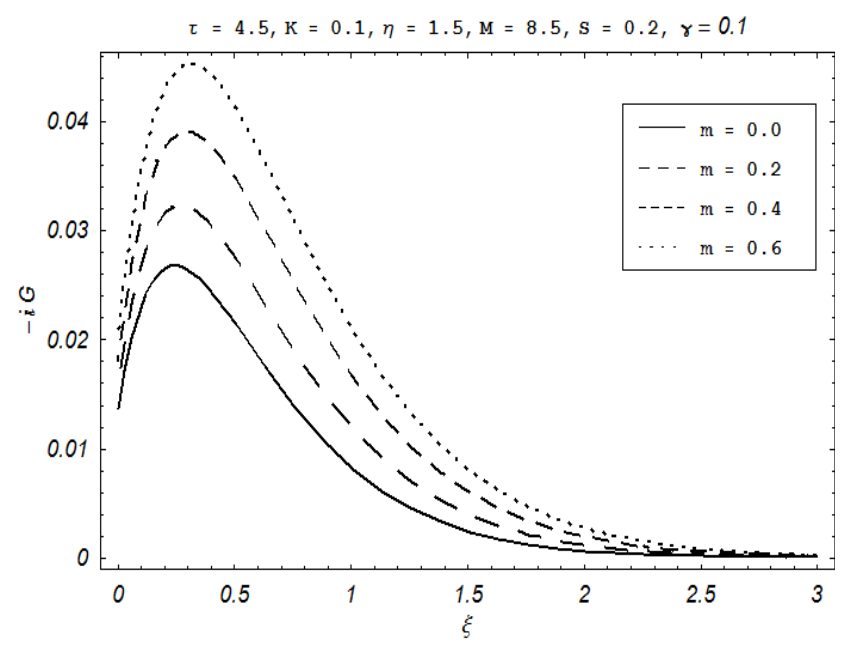

(b)

Figure 3. Profiles of velocity for different values of $m$.

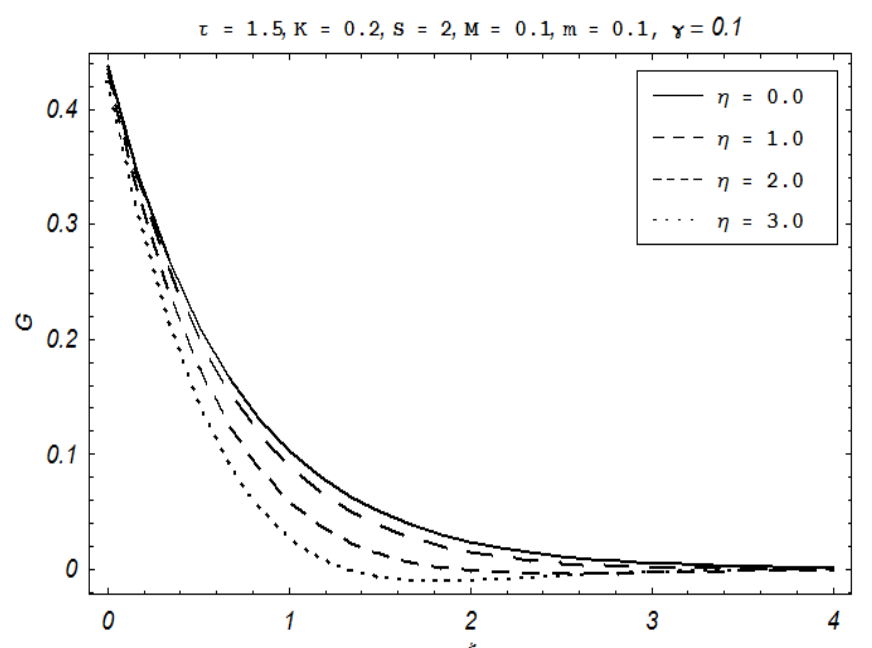

(a)

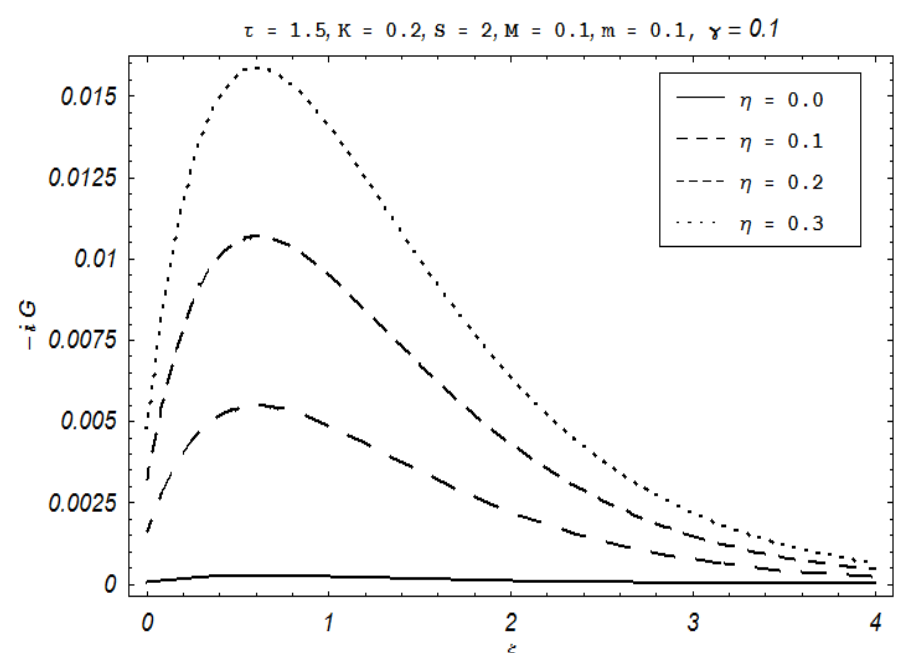

(b)

Figure 4. Profiles of velocity for different values of $\eta$.

there is no suction $S=0$, the fluid velocity is minimum and then increasing with increasing values of $S$. The variation of velocity profiles for the injection case is quite opposite to that of suction case as shown in Figure 6. Further, Figure 7 illustrates the profiles of the complex velocity for various values of the slip parameter $\gamma$. It is interesting to note that when $\gamma=0$, which corresponds to the no slip condition, the value of the real part of velocity is 1 while the imaginary part of velocity is 0 . This shows a good match with the boundary condition given by Equation 16; also it ensures the accuracy of the results displayed here. It is further noted that for large values of $\gamma$, velocity of the real and imaginary parts are decreasing. Finally, Figure 8 shows variation in the velocity profile for different values of dimensionless time $\tau$ for fixed values of other parameters. It is noticed that for large values of $\tau$, both the real and imaginary parts of velocity are increasing in the vicinity of the plate and then decrease smoothly, finally approaches to zero for larger values of the span wise coordinate $\xi$. Moreover, it is further observed that for each value of $\tau$ the boundary layer thickness for the imaginary part of velocity is greater 


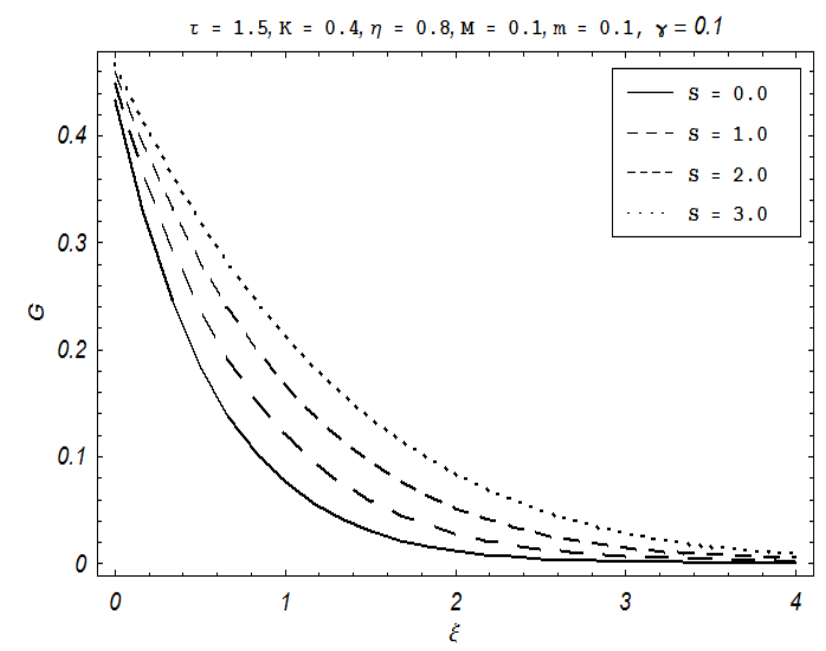

(a)

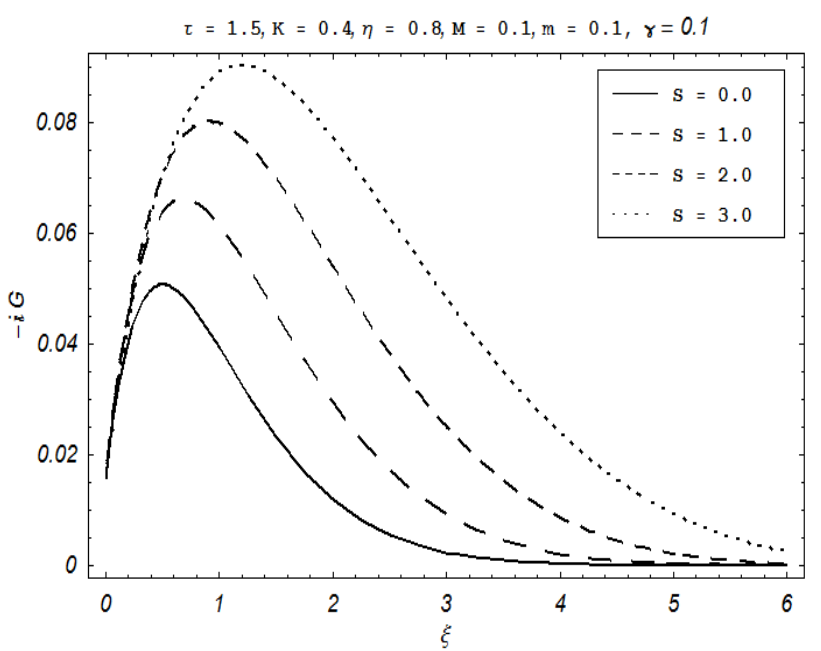

(b)

Figure 5. Profiles of velocity for different values of $S \geq 0$.

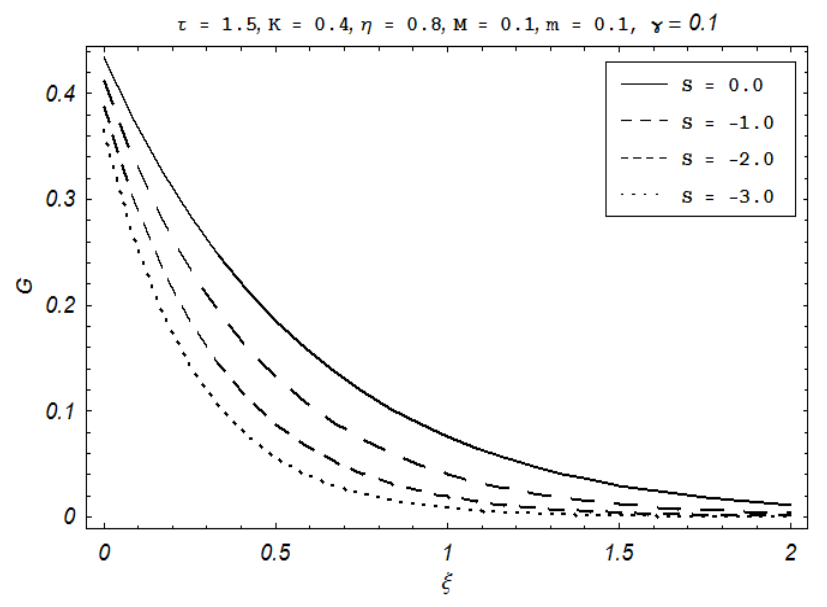

(a)

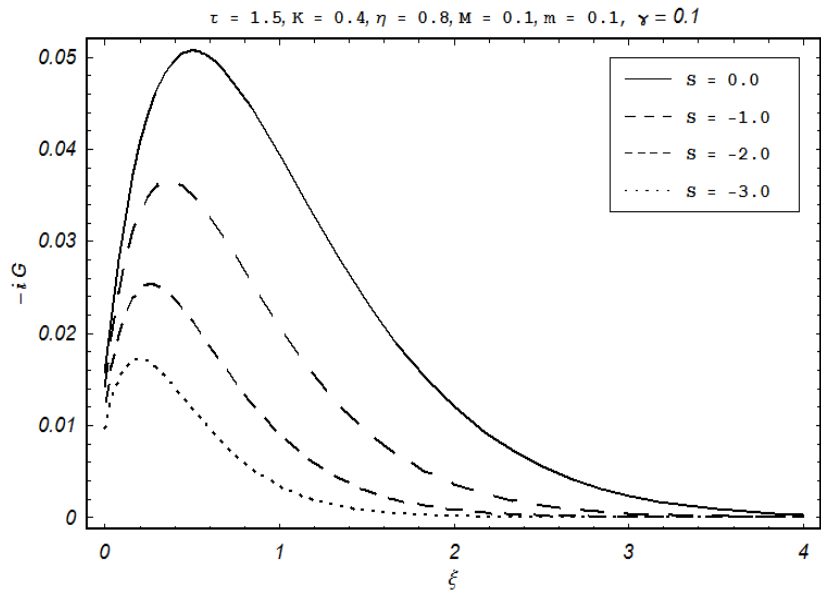

(b)

Figure 6. Profiles of velocity for different values of $S \leq 0$.

than the real part of velocity.

\section{CONCLUSIONS}

In this paper, we have examined the slip and Hall effects on the unsteady hydromagnetic rotating flow of viscous fluid past a suddenly moved plate with uniform suction $S>0$ or injection $S<0$. The modeling was done in a porous space. Employing Laplace transform technique, the governing equation was solved for the closed form analytical solution in the complex plane. The flow

characteristics are illustrated using graphs for both real and imaginary parts of the complex velocity to see the effects of various embedded flow parameters. The following conclusions are extracted from this study:

1) The velocity field decreases for larger values of magnetic parameter.

2) An increase in permeability parameter increases the fluid velocity.

3) The velocity increases for higher values of Hall parameter.

4) With increasing values of rotation parameter, the real 


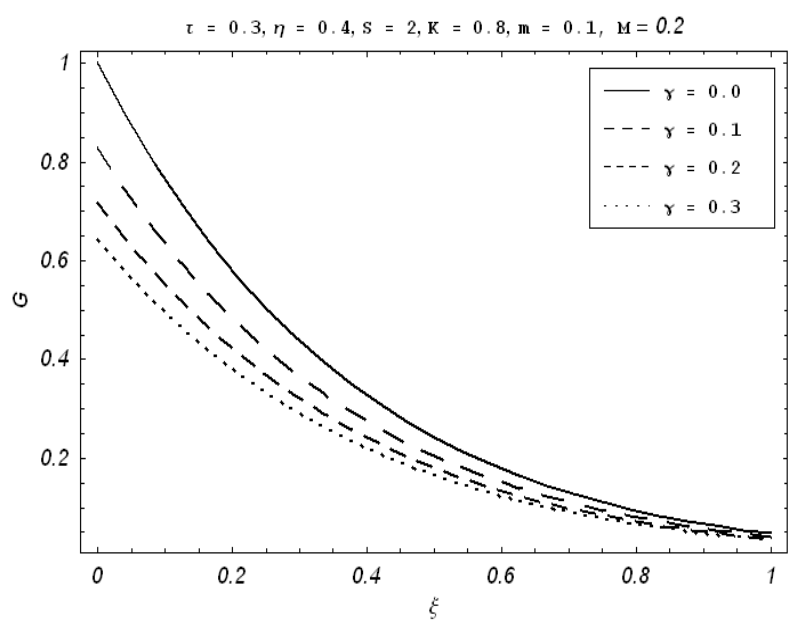

(a)

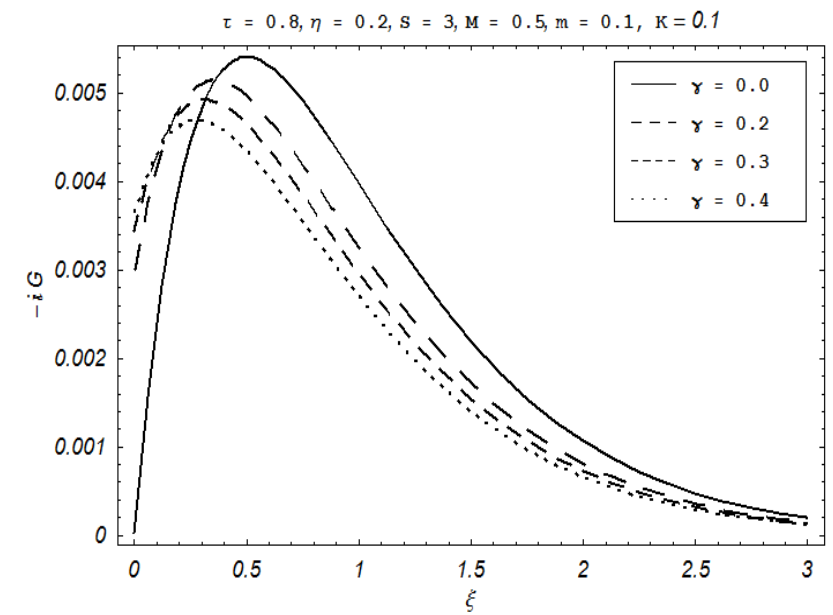

(b)

Figure 7. Profiles of velocity for different values of $\gamma$.

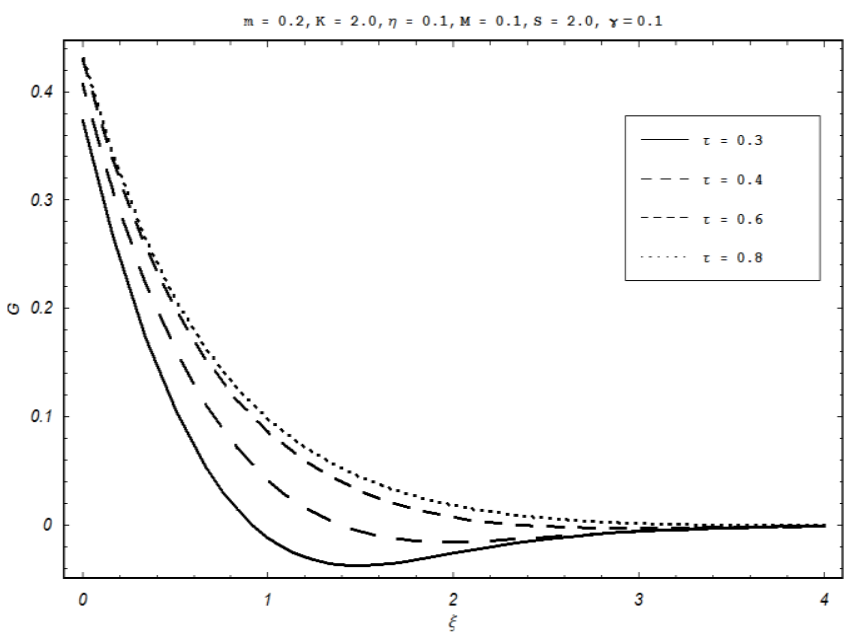

(a)

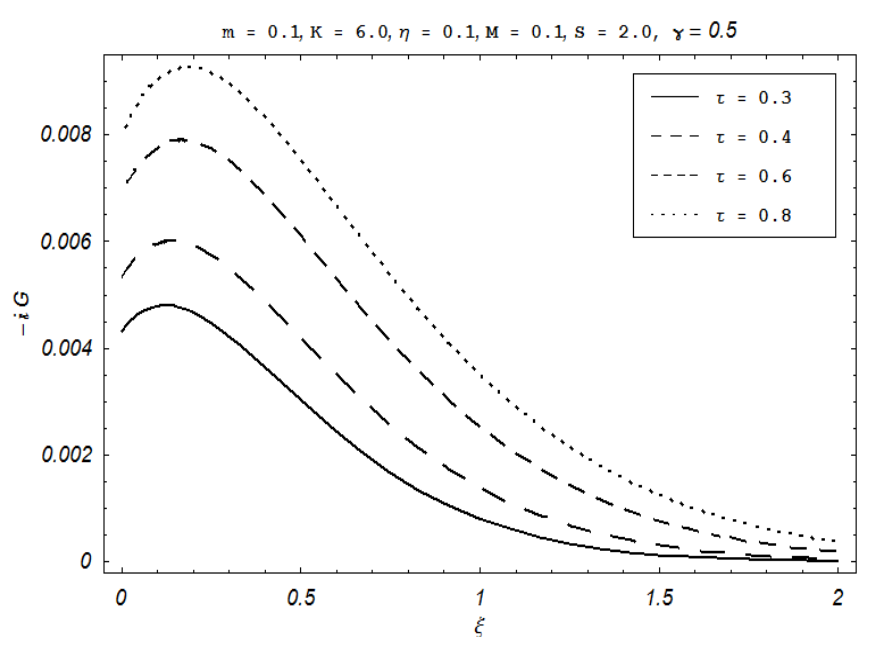

(b)

Figure 8. Profiles of velocity for different values of $\tau$.

part of velocity is decreasing whereas the magnitude of the imaginary part is increasing.

5) An increase in suction parameter increases the complex velocity of the fluid while injection parameter decreases it.

6) When the slip parameter is increased, the velocity field is decreased.

7) The condition for no slip is recovered when $\gamma=0$.

8) The complex velocity is an increasing function of time. 10) In the absence of magnetic field and slip condition, the results of Jana et al. (2010) can be obtained as a special case.

\section{ACKNOWLEDGEMENT}

The authors would like to acknowledge the Research Management Centre -- UTM for the financial support through vote number $\mathrm{S} 78528$ and $4 \mathrm{~F} 019$ for this research.

\section{REFERENCES}

Abelman S, Momoniat E, Hayat T (2009). Couette flow of a third grade fluid with rotating frame and slip condition. Non-Linear Anal. Real World Appl., 10: 3329-3334. 
Asghar S, Hanif K, Hayat T (2007). The effect of the slip condition on unsteady flow due to non-coaxial rotations of disk and a fluid at infinity. Meccanica, 42: 141-148.

Blake TD (1990). Slip between a liquid and a solid: D.M. Tolstoi's (1952) theory reconsidered. Colloids. Surfaces, 47: 135-145.

Chawla SS, Srivastava PK, Gupta AS (2009). Rotationally symmetric flow over a rotating disk. Int. J. Non-Linear Mech., 44: 717-726.

Gosh SK (1993). Unsteady hydromagnetic flow in a rotating channel with oscillating pressure gradient. J. Phys. Soc. Jpn, 62: 3893-3903.

Gosh SK (1999). Hall effect on unsteady hydromagnetic flow in rotating channel permeated by an inclined magnetic field in the presence of an oscillator, Czechoslovak J. Phys., 49: 465-472.

Hayat T, Fetecau C, Sajid M (2008a). Analytic solution for MHD Transient rotating flow of a second grade fluid in a porous space. Non-Linear Anal. Real World Appl., 9: 16191627.

Hayat T, Fetecau C, Sajid M (2008b). On MHD transient flow of a Maxwell fluid in a porous medium and rotating frame, Phys. Lett. A, 372: 1639-1644.

Hayat T, Nawaz M (2011a). Unsteady stagnation point flow of viscous fluid caused by an impulsively rotating disk. J. Taiwan Inst. Chem. Eng., 42: 41-49.

Hayat T, Najam S, Khalique CM (2010a). Couette flow of an Oldroyd-B fluid with slip condition. J. Porous Media, 13: 999-1006.

Hayat T, Najam S, Asghar S (2010b). Rotating flow of a generalized Burgers' fluid with slip condition. J. Porous. Media, 13: 839-845.

Hayat T, Khan SB, Khan M (2007). The influence of Hall current on the rotating oscillating flows of an Oldroyd-B fluid in a porous medium. Non-Linear Dyn, 47: 353-362.

Hayat T, Qasim M, Mesloub S (2011b). MHD flow and heat transfer over permeable stretching sheet with slip conditions. Int. J. Numerical Methods in Fluids, 66: 963-975.

Jana M, Maji SL, Das S, Jana RN (2010). Unsteady flow of viscous fluid through a porous medium bounded by a porous plate in a rotating system. J. Porous. Media, 13: 645-653.

Jana, RN, Datta N, Mazumder BS (1977). Magnetohydrodynamic Couette flow and heat transfer in a rotating system. J. Phys. Soc. Jpn, 42: 1034-1039.

Jha BK, Apere CA (2010). Hall and ion-slip effects on unsteady MHD Couette flow in a rotating system with suction and injection. J. Phys. Soc. Jpn, 79: 104401(1-9).

Katagiri $M$ (1962). Flow formation in Couette motion in magnethohydrodynamics. J. Phys. Soc. Jpn, 17: 393-396.

Makinde OD, Mhone PY (2005). Heat transfer to MHD oscillatory flow in a channel filled with porous medium. Rom. J. Phys., 50: 931-938.

Mandal G, Mandal KK (1983). Effects of Hall current on MHD Couette flow between thick arbitrarily conducting plates in a rotating system. J. Phys. Soc. Jpn, 52: 470-477.
Mehmood A, Ali A (2007). The effect of slip condition on unsteady MHD oscillatory flow of a viscous fluid in a planer channel. Rom. J. Phys., 52: 85-91.

Navier CLMH (1823). Mem.Acad.Sci. Inst. France. 1: 414-416.

Pit $R$, Hervet $H$, Leger $L$ (1999). Friction and slip of a simple liquid at a solid surface.TribologyLett., 7: 147-152.

Qasim M, Hayat T, Hendi AA (2011). Effects of slip conditions on stretching flow with ohmic dissipation and thermal radiation. Heat Transfer: Asian Research. Heat Transfer Asian Research. 40: 641654.

Sahoo SN, Panda JP, Dash GC (2010). Hydromagnetic oscillatory flow and heat transfer of a viscous liquid past a vertical porous plate in a rotating medium. Indian J. Sci. Technol., 3: 818-822.

Seth GS, Ansari MS, Nandkeolyar R (2010). Unsteady hydromagnetic Couette flow within porous plates in a rotating system. Adv. Appl. Math. Mech., 2: 286-302.

Singh AK, Singh NP, Singh U, Singh H (2009). Convective flow past an accelerated porous plate in rotating system in presence of magnetic field.Int. J. Heat Mass. Transfer., 52: 3390-3395.

Turkyilmazoglu M (2009). Exact solutions for the incompressible viscous fluid of a porous rotating disk flow. Int. J. Non-Linear. Mech., 44: 352-357.

Vinogradova OI (1995). Drainage of a thin film confined between hydrophobic surfaces. Langmuir, 11: 2213-2220.

Zhu Y, Granick S (2002). Limits of the hydrodynamic no-slip liquid flow boundary condition. Phys. Rev. Lett., 88: 106102 (1-4). 